\title{
Iterative Learning Control of Upper Limb Reaching using Functional Electrical Stimulation *
}

\author{
C. T. Freeman, I. L. Davies, P. L. Lewin and E. Rogers \\ School of Electronics and Computer Science, \\ University of Southampton, Southampton, SO17 1BJ, UK \\ (e-mail: cf@ecs.soton.ac.uk)
}

\begin{abstract}
An iterative learning control scheme for the application of functional electrical stimulation to the human arm is designed and implemented. The task is to track trajectories in the horizontal plane and stimulation is applied to the triceps muscle. A model of the arm is first derived which includes assistive torque about the shoulder provided by a robotic arm. A linearising controller is then designed and a linear ILC algorithm is applied to the resulting system. Experimental results show that a high level of performance can be achieved in practice, and provide justification for the system to be subsequently used by stroke patients for rehabilitation.
\end{abstract}

\section{INTRODUCTION}

Strokes affect between 174 and 216 people per 100,000 population in the UK each year, and half of all acute stroke patients starting rehabilitation will have a marked impairment of function in one arm (Mant et al. [2004]). Functional electrical stimulation (FES) can provide the experience of moving for the patient, which is necessary if sensory-motor function is to be regained. Recent studies have shown that when stimulation is associated with a voluntary attempt to move the limb, improvement is enhanced (Burridge and Ladouceur [2001]). Open-loop methods for the control of FES (see, for example, Davoodi and Andrews [2004]) have not provided the high level of performance necessary to fully promote this association. Closedloop and model-based schemes, however, have overwhelmingly concentrated on the lower rather than the upper limb. Neural networks are one of the few approaches that have successfully been used to control FES applied to the arm, but these require extensive training and have unresolved stability issues due to their black-box structure (see Lan et al. [1994]).

An experimental test facility incorporating a five-link planar robotic arm and an overhead trajectory projection system (see Freeman et al. [2007] for details) has been developed in order to provide a controlled environment in which to apply electrical stimulation to stroke patients. The subject is seated with their arm strapped to the robot, and the task presented to them is to repeatedly track a number of reaching trajectories using a combination of voluntary control and surface FES applied to muscles in their impaired shoulder and arm. The electrical stimulation is mediated using iterative learning control (ILC), a technique that is applicable to systems operating in a cyclical mode. This is one of the few advanced control techniques which has previously been applied to stimulation of the upper limb, although a high level of performance has not been achieved in practice (Dou et al. [1999]). Given the nature of the task, ILC is an obvious choice but also provides a framework which is capable of producing accurate tracking provided that the difference in voluntary effort (interpreted as an external disturbance

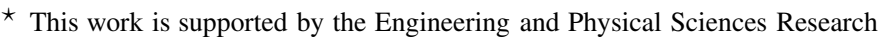
Council (EPSRC). Grant no. EP/C51873X/1.
}

applied to the system) is sufficiently small from one trial to the next (see, for example, Moore [1992]). When accurate tracking of the trajectory is achieved, the stimulation will be reduced in order to promote sustained voluntary effort by the subject.

In this paper ILC is used to control the FES applied to the triceps of an unimpaired subject who provides no voluntary effort. The robot supplies an assistive torque about the shoulder to allow full reaching tasks to be accomplished that are driven by the stimulation. The results show that high performance can be achieved, and confirm the efficacy of the system prior to its use by stroke patients.

\section{WORKSTATION DESCRIPTION}

The robotic workstation consists of a five-link planar robotic arm rigidly connected to an overhead projection system, and is shown in Figure 1. A subject is strapped to the extreme link and

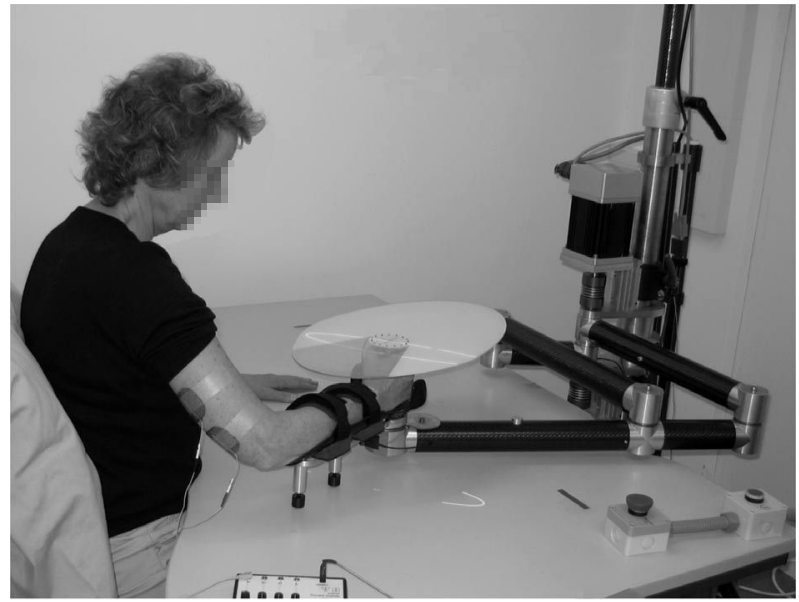

Fig. 1. Unimpaired subject using the robotic workstation.

a 6 axis force/torque sensor records the force they apply to the robotic end effector. The robotic arm is used to constrain the subject's arm, to impose forces on the end-effector that make the task feel 'natural' to the subject, and to apply assistance during the performance of tracking tasks. The stroke patient's 
task during the treatment will be to track a range of trajectories that are projected onto a target mounted above their hand.

\section{HUMAN ARM MODEL}

The mathematical description of the subject's arm consists of a model of the passive dynamical system to which the torque generating properties of the stimulated muscle is then added.

\subsection{Passive System}

Figure 2 shows the geometry of the constrained human arm model. The first link represents the upper arm, from the acromion to the elbow, with length $\left(l_{u 1}+l_{u 2}\right)$. The second link represents the forearm, from the elbow to the thumb web, with length $\left(l_{f 1}+l_{f 2}\right)$. The constraint means that the forearm must lie in the horizontal plane, and rotation is possible about the axis along the upper arm. The point, $Q$, denotes where the subject's hand grasps the robot, and components of the forces applied in the $\boldsymbol{x}_{0}$ and $\boldsymbol{y}_{0}$ directions are denoted by $F_{x_{0}}$ and $F_{y_{0}}$ respectively. Actuation is provided by the triceps, which has been modelled as supplying a torque, $T_{\beta} \geq 0$, acting about an axis orthogonal to both the upper arm and forearm. To satisfy the horizontal

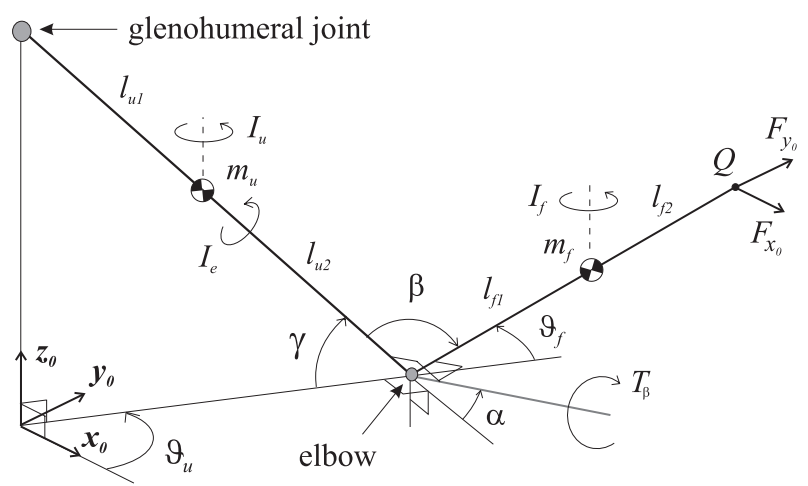

Fig. 2. Geometry of constrained human arm.

constraint it is necessary to set

$$
\alpha\left(\vartheta_{f}\right)=\arccos \left(\frac{c_{f} s_{\gamma}}{\sqrt{1-c_{f}^{2} c_{\gamma}^{2}}}\right)
$$

which corresponds to an elbow angle of

$$
\beta\left(\vartheta_{f}\right)=\arccos \left(-c_{f} c_{\gamma}\right)
$$

Here $c_{f}$ and $c_{\gamma}$ denote $\cos \left(\vartheta_{f}\right)$ and $\cos (\gamma)$ respectively, and $c_{u}$ and $c_{u f}$ will be used to denote $\cos \left(\vartheta_{u}\right)$ and $\cos \left(\vartheta_{u}+\vartheta_{f}\right)$. Similar expressions are used for the case of $\sin (\cdot)$. The unitary axis about which $T_{\beta}$ is applied is given by

$$
\frac{1}{\sqrt{1-c_{f}^{2} c_{\gamma}^{2}}}\left[\begin{array}{c}
-s_{f} s_{\gamma} \\
c_{f} s_{\gamma} \\
-s_{f} c_{\gamma}
\end{array}\right]
$$

The dynamic model of the constrained arm can then be expressed in the form

$$
\begin{aligned}
& \boldsymbol{B}(\boldsymbol{q}) \ddot{\boldsymbol{q}}+\boldsymbol{C}(\boldsymbol{q}, \dot{\boldsymbol{q}}) \dot{\boldsymbol{q}}+\boldsymbol{F}(\boldsymbol{q}, \dot{\boldsymbol{q}})=\boldsymbol{\tau}-\boldsymbol{J}^{T}(\boldsymbol{q}) \boldsymbol{h} \\
& \text { where } \boldsymbol{q}=\left[\vartheta_{u} \vartheta_{f}\right]^{T}, \boldsymbol{\tau}=\left[0 T_{\beta} \frac{-s_{f} c_{\gamma}}{\sqrt{1-c_{f}^{2} c_{\gamma}^{2}}}\right]^{T}, \boldsymbol{h}=\left[F_{x_{0}} F_{y_{0}}\right]^{T} \text { and } \\
& \boldsymbol{B}(\boldsymbol{q})=\left[\begin{array}{l}
b_{1} b_{2} \\
b_{2} b_{3}
\end{array}\right], \boldsymbol{C}(\boldsymbol{q}, \dot{\boldsymbol{q}})=\left[\begin{array}{cc}
-2 c_{1} \dot{\vartheta}_{f}-c_{1} \dot{\vartheta}_{f} \\
c_{1} \dot{\vartheta}_{u} & c_{2} \dot{\vartheta}_{f}
\end{array}\right], \\
& \boldsymbol{J}^{T}(\boldsymbol{q})=\left[\begin{array}{cc}
-\left(l_{u 1}+l_{u 2}\right) c_{\gamma} s_{u}-\left(l_{f 1}+l_{f 2}\right) s_{u f} & \left(l_{u 1}+l_{u 2}\right) c_{\gamma} c_{u}+\left(l_{f 1}+l_{f 2}\right) c_{u f} \\
-\left(l_{f 1}+l_{f 2}\right) s_{u f} & \left(l_{f 1}+l_{f 2}\right) c_{u f}
\end{array}\right]
\end{aligned}
$$

with

$$
\begin{aligned}
& b_{1}=m_{u}\left(l_{u 1} c_{\gamma}\right)^{2}+I_{u}+m_{f}\left(l_{f 1}^{2}+\left(\left(l_{u 1}+l_{u 2}\right) c_{\gamma}\right)^{2}+2\left(l_{u 1}+l_{u 2}\right) c_{\gamma} l_{f 1} c_{f}\right)+I_{f} \\
& b_{2}=m_{f}\left(l_{f 1}^{2}+\left(l_{u 1}+l_{u 2}\right) c_{\gamma} l_{f 1} c_{f}\right)+I_{f}, \quad b_{3}=m_{f} l_{f 1}^{2}+I_{f}+I_{e}\left(\frac{s_{\gamma}}{1-c_{f}^{2} c_{\gamma}^{2}}\right)^{2} \\
& c_{1}=m_{f}\left(l_{u 1}+l_{u 2}\right) c_{\gamma} l_{f 1} s_{f}, \quad c_{2}=-2 I_{e}\left(\frac{s_{\gamma}^{2} c_{\gamma}^{2} c_{f} s_{f}}{\left(1-c_{f}^{2} c_{\gamma}^{2}\right)^{3}}\right)
\end{aligned}
$$

The most general form of the friction term considered is

$$
\boldsymbol{F}(\boldsymbol{q}, \dot{\boldsymbol{q}})=\left[F_{1}\left(\vartheta_{u}, \dot{\vartheta}_{u}\right) F_{2}\left(\vartheta_{f}, \dot{\vartheta}_{f}\right)\right]^{T}
$$

in which $F_{1}(\cdot)$ and $F_{2}(\cdot)$ are piecewise linear functions.

A form of impedance control (see Colgate and Hogan [1988]) is used to control the robotic arm, which results in the relationship

$$
-\boldsymbol{h}=\boldsymbol{K}_{K_{x}} \tilde{\boldsymbol{x}}-\boldsymbol{K}_{B_{x}} \dot{\boldsymbol{x}}-\boldsymbol{K}_{M_{x}} \ddot{\boldsymbol{x}}
$$

at $Q$, where $\hat{x}$ is the reference position, $\tilde{x}=\hat{x}-\boldsymbol{x}, \boldsymbol{x}=\boldsymbol{k}(\boldsymbol{q})$, $\dot{\boldsymbol{x}}=\boldsymbol{J}(\boldsymbol{q}) \dot{\boldsymbol{q}}$ and $\ddot{\boldsymbol{x}}=\boldsymbol{J}(\boldsymbol{q}) \ddot{\boldsymbol{q}}+\dot{\boldsymbol{J}}(\boldsymbol{q}, \dot{\boldsymbol{q}}) \dot{\boldsymbol{q}}$. Here $\boldsymbol{x}=\boldsymbol{k}(\boldsymbol{q})$ is the direct kinematics equation for the human arm system. When the robot is moved freely by the subject in the absence of assistance, the gain matrices are set as $\boldsymbol{K}_{K_{x}}=\mathbf{0}, \boldsymbol{K}_{B_{x}}=K_{B_{x}} \boldsymbol{I}$ and $\boldsymbol{K}_{M_{x}}=K_{M_{x}} \boldsymbol{I}$. The values of $K_{B_{x}}$ and $K_{M_{x}}$ assume positive values and are tuned to create a 'natural' feel. When the robot is required to move the subject's arm along predefined trajectories, it is necessary to set $\boldsymbol{K}_{K_{x}}=K_{K_{x}} \boldsymbol{I}$ with $K_{K_{x}}>0$. The three gains are then tuned to produce the required tracking performance. The form of the gain matrices for the case where the robot applies assistance during tracking tasks, is described in Section 4. Further details of the robotic controller are given in (Freeman et al. [2007]).

\subsection{Muscle Model}

A model of the torque, $T_{\beta}$, generated by electrically stimulated muscle acting about a single joint is given by

$$
T_{\beta}(\beta, \dot{\beta}, u, t)=g(u, t) \times F_{a}(\beta, \dot{\beta})+F_{p}(\beta, \dot{\beta})
$$

where $u$ denotes the stimulation pulsewidth applied, and $\beta$ is the joint angle (see Shue et al. [1995]). A Hammerstein structure incorporating a static non-linearity, $h_{I R C}(u)$, representing the isometric recruitment curve, cascaded with linear activation dynamics, $h_{L A D}(t)$, produces the first term, $g(u, t)$. The activation dynamics can be modelled as a critically damped second order system (Baratta and Solomonow [1990]). The term $F_{a}(\beta, \dot{\beta})$ describes the multiplicative effect of the joint angle and joint angular velocity on the active torque developed by the muscle. The term $F_{p}(\beta, \dot{\beta})$ accounts for the passive properties of the joint. Since $\gamma$ is invariant, (2) means it is accounted for when using the frictional form of (7). A full description of the procedures used to establish the parameters appearing in the model, and also identification results for the subject whose results appear in Section 6, is given in (Freeman et al. [2008]).

\section{ROBOTIC ASSISTANCE AND TRAJECTORY CHOICE}

The action of the robotic arm must now be considered in order to make the task a feasible yet productive one. The following points therefore concern the choice of trajectory and necessary role of the robot during the performing of the task:

(1) The trajectories will be elliptical reaching tasks for the subject's dominant arm, and should be achievable given their identified arm model.

(2) The triceps muscle will provide the sole actuating torque about the elbow, and the robotic arm will use the control scheme given by (8) to make the dynamics about the elbow feel 'natural' to the subject. 
(3) The robotic arm will provide a torque acting about the subject's shoulder in order to track the reference in a manner which is entirely governed by the angle of the forearm. This then makes the task feasible without lessening the role played by the triceps.

Combining (8) and (4) results in

$\boldsymbol{B}(\boldsymbol{q}) \ddot{\boldsymbol{q}}+\boldsymbol{C}(\boldsymbol{q}, \dot{\boldsymbol{q}}) \dot{\boldsymbol{q}}+\boldsymbol{F}(\boldsymbol{q}, \dot{\boldsymbol{q}})=\boldsymbol{\tau}+\boldsymbol{J}^{T}(\boldsymbol{q})\left(\boldsymbol{K}_{K_{x}} \tilde{\boldsymbol{x}}-\boldsymbol{K}_{B_{x}} \dot{\boldsymbol{x}}-\boldsymbol{K}_{M_{x}} \ddot{\boldsymbol{x}}\right)$

To separate the dynamics of the end-effector in the directions corresponding to the human arm joint angles, it is then necessary to set

$$
\boldsymbol{K}_{K_{x}} \tilde{\boldsymbol{x}}-\boldsymbol{K}_{B_{x}} \dot{\boldsymbol{x}}-\boldsymbol{K}_{M_{x}} \ddot{\boldsymbol{x}}=\boldsymbol{J}^{-T}(\boldsymbol{q})\left(\boldsymbol{K}_{K_{q}} \tilde{\boldsymbol{q}}-\boldsymbol{K}_{B_{q}} \dot{\boldsymbol{q}}-\boldsymbol{K}_{M_{q}} \ddot{\boldsymbol{q}}\right)
$$

where $\tilde{\boldsymbol{q}}=\hat{\boldsymbol{q}}-\boldsymbol{q}$ and $\hat{\boldsymbol{q}}=\boldsymbol{k}^{-1}(\hat{\boldsymbol{x}})$. To meet the requirements of points 2) and 3) above, it is then required that

$$
\begin{gathered}
\boldsymbol{K}_{K_{q}}=\operatorname{diag}\left\{K_{K_{1}}, 0\right\}, \boldsymbol{K}_{B_{q}}=\operatorname{diag}\left\{K_{B_{1}}, K_{B_{2}}\right\} \\
\boldsymbol{K}_{M_{q}}=\operatorname{diag}\left\{K_{M_{1}}, K_{M_{2}}\right\}
\end{gathered}
$$

and $\hat{\boldsymbol{q}}=\left[\hat{\vartheta}_{u} \hat{\vartheta}_{f}\right]^{T}$ where $K_{K_{1}}, K_{B_{1}}, K_{B_{2}}, K_{M_{1}}, K_{M_{2}} \geq 0$. This allows a choice of arbitrary second order dynamics to be imposed about the shoulder and the damping and inertia about the elbow to be prescribed. This produces the expression

$$
\boldsymbol{\tau}+\left[\begin{array}{c}
K_{K_{1}} \tilde{\vartheta}_{u}-K_{B_{1}} \dot{\vartheta}_{u}-K_{M_{1}} \ddot{\vartheta}_{u} \\
-K_{B_{2}} \dot{\vartheta}_{f}-K_{M_{2}} \ddot{\vartheta}_{f}
\end{array}\right]
$$

for the right-hand side of (10), and provides the necessary dynamic relationship for both components of the torque. To arrive at the required values of $\hat{\vartheta}_{u}$ and $\hat{\vartheta}_{f}$, components of (11) are compared to give

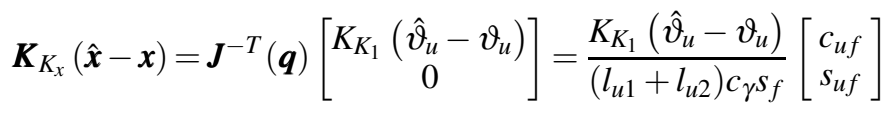

This leads to a solution

$$
\boldsymbol{K}_{K_{x}}=\frac{K_{K_{1}}\left(\hat{\vartheta}_{u}-\vartheta_{u}\right)}{|\hat{\boldsymbol{x}}-\boldsymbol{x}|\left(l_{u 1}+l_{u 2}\right) c_{\gamma} \boldsymbol{s}_{f}} \boldsymbol{I}, \quad \hat{\boldsymbol{x}}=\boldsymbol{x}+|\hat{\boldsymbol{x}}-\boldsymbol{x}|\left[\begin{array}{c}
c_{u f} \\
s_{u f}
\end{array}\right]
$$

so that $\hat{\boldsymbol{x}}$ is a point lying on a line extending along the forearm and passing through $\boldsymbol{x}$. To achieve the tracking task it must therefore be set equal to the point of intersection with the trajectory. This is shown in Figure 3, in which $\boldsymbol{x}^{*}(t)=\boldsymbol{k}\left(\boldsymbol{q}^{*}(t)\right)$ with $\boldsymbol{q}^{*}(t)$ defined in (16). The remaining robotic controller

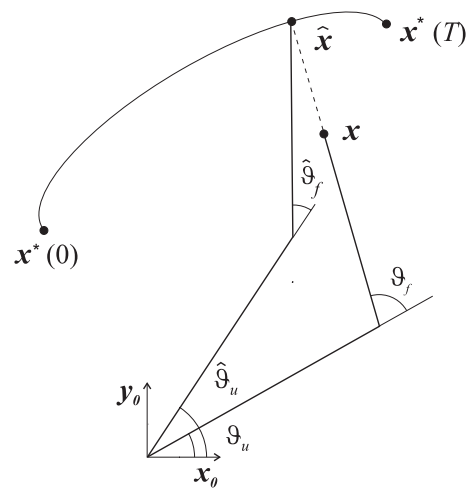

Fig. 3. Trajectory Geometry.

matrices may be chosen to satisfy

$$
\left\{\begin{array}{l}
\boldsymbol{K}_{B_{x}}=\boldsymbol{J}^{-T}(\boldsymbol{q}) \boldsymbol{K}_{B_{q}} \boldsymbol{J}^{-1}(\boldsymbol{q}) \\
\boldsymbol{K}_{M_{x}}(\boldsymbol{J}(\boldsymbol{q}) \ddot{\boldsymbol{q}}+\dot{\boldsymbol{J}}(\boldsymbol{q}, \dot{\boldsymbol{q}}) \dot{\boldsymbol{q}})=\boldsymbol{J}^{-T}(\boldsymbol{q}) \boldsymbol{K}_{M_{q}}
\end{array}\right.
$$

with the gains given by (12). Let the trajectory be defined by

$$
\boldsymbol{q}^{*}(t)=\left[\vartheta_{u}^{*}(t) \vartheta_{f}^{*}(t)\right]^{T}, \quad t \in[0 T]
$$

Eliminating $t$ from the components provides the relationship $\vartheta_{u}=\Psi\left(\vartheta_{f}\right)$. The reference point is then defined formally as $\hat{\boldsymbol{x}}=\boldsymbol{\Omega}(\boldsymbol{x}, \Psi(\cdot)): \boldsymbol{k}\left(\left[\begin{array}{c}\Psi\left(\hat{\vartheta}_{f}\right) \\ \hat{\vartheta}_{f}\end{array}\right]\right) \mid \boldsymbol{k}\left(\left[\begin{array}{c}\Psi\left(\hat{\vartheta}_{f}\right) \\ \hat{\vartheta}_{f}\end{array}\right]\right)=\boldsymbol{x}+\lambda\left[\begin{array}{c}c_{u f} \\ s_{u f}\end{array}\right]$ where $\lambda$ is a scalar. The complete control system is shown in Figure 4. The gain (14) can then be written explicitly as

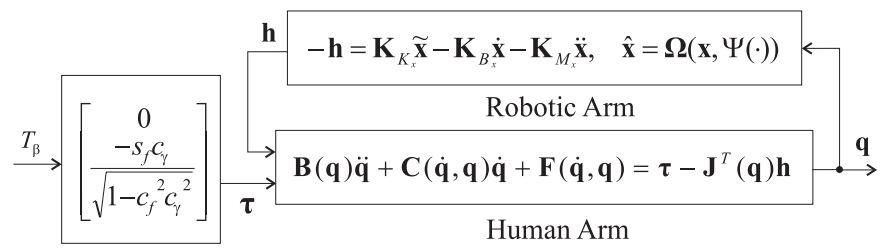

Fig. 4. Human arm system with robotic assistance.

$$
K_{K_{x}}(\boldsymbol{x}, \Psi(\cdot))=\frac{K_{K_{1}}\left(\Psi\left(\hat{\vartheta}_{f}\right)-\vartheta_{u}\right)}{\lambda\left(l_{u 1}+l_{u 2}\right) c_{\gamma} s_{f}} \boldsymbol{I}
$$

Certain points will now be made concerning the nature of the trajectories and the selection of $K_{B_{2}}$ and $K_{M_{2}}$ that govern how the task feels to the subject:

(1) For $\Psi\left(\vartheta_{f}\right)$ to be a one-one continuous function, both $\vartheta_{u}^{*}(t)$ and $\vartheta_{f}^{*}(t)$ must be monotone.

(2) The bottom row of (10), with the expressions given by (8) and (11), and the control gains (12), is

$$
\begin{array}{r}
{\left[\begin{array}{ll}
b_{2} b_{3}
\end{array}\right]\left[\begin{array}{l}
\ddot{\vartheta}_{u} \\
\ddot{\vartheta}_{f}
\end{array}\right]+\left[c_{1} \dot{\vartheta}_{u} c_{2} \dot{\vartheta}_{u}\right]\left[\begin{array}{c}
\dot{\vartheta}_{u} \\
\dot{\vartheta}_{f}
\end{array}\right]+F_{2}\left(\vartheta_{f}, \dot{\vartheta}_{f}\right)} \\
=T_{\beta}\left(\frac{-s_{f} c_{\gamma}}{\sqrt{1-c_{f}^{2} c_{\gamma}^{2}}}\right)-K_{B_{2}} \dot{\vartheta}_{f}-K_{M_{2}} \ddot{\vartheta}_{f}
\end{array}
$$

If the robotic assistance system provides perfect tracking of $\hat{\vartheta}_{u}$ by $\vartheta_{u}$, so that $\vartheta_{u}=\Psi\left(\vartheta_{f}\right)$, then $\dot{\vartheta}_{u}=\dot{\vartheta}_{f} \Psi^{\prime}\left(\vartheta_{f}\right)$ and $\ddot{\vartheta}_{u}=$ $\ddot{\vartheta}_{f} \Psi^{\prime}\left(\vartheta_{f}\right)+\dot{\vartheta}_{f}^{2} \Psi^{\prime \prime}\left(\vartheta_{f}\right)$. The system is then independent of $\vartheta_{u}$, and can be written solely in terms of $\vartheta_{f}$, giving

$$
\begin{aligned}
& T_{\beta}=\left(\frac{-\sqrt{1-c_{f}^{2} c_{\gamma}^{2}}}{s_{f} c_{\gamma}}\right)\left\{K_{B_{2}} \dot{\vartheta}_{f}+\left(K_{M_{2}}+b_{3}+b_{2} \Psi^{\prime}\left(\vartheta_{f}\right)\right) \ddot{\vartheta}_{f}\right. \\
& \left.+\left(b_{2} \Psi^{\prime \prime}\left(\vartheta_{f}\right)+c_{1} \Psi^{\prime}\left(\vartheta_{f}\right)^{2}+c_{2} \Psi^{\prime}\left(\vartheta_{f}\right)\right) \dot{\vartheta}_{f}^{2}+F_{2}\left(\vartheta_{f}, \dot{\vartheta}_{f}\right)\right\}
\end{aligned}
$$

(3) In practice $c_{a 1}, c_{a 2} \ll b_{a 2}, b_{a 3}$ and $I_{e} \approx 0.005 \mathrm{Kgm}^{2}$ (see Freeman et al. [2008] for examples of identified values), and the trajectories are chosen so that the derivatives of $\Psi(\cdot)$ are small. Therefore $T_{\beta}$ can be approximated as

$$
\left(\frac{-\sqrt{1-c_{f}^{2} c_{\gamma}^{2}}}{s_{f} c_{\gamma}}\right)\left\{K_{B_{2}} \dot{\vartheta}_{f}+\left(K_{M_{2}}+b_{a 3}\right) \ddot{\vartheta}_{f}+F_{a 2}\left(\vartheta_{f}, \dot{\vartheta}_{f}\right)\right\}
$$

which is shown schematically in Figure 5.

(4) In practice the existence of a torque that will allow $\vartheta_{f}^{*}(t)$ to be tracked perfectly is ensured by selecting slow trajectories that comprise half ellipse segments. The start and end points are chosen so that they can be reached by a smooth extension about the elbow. The gains, $K_{B_{2}}$ and $K_{M_{2}}$, are selected in order to mimic a realistic activity, and their effect on the overall control scheme is discussed in Section 5.1. 


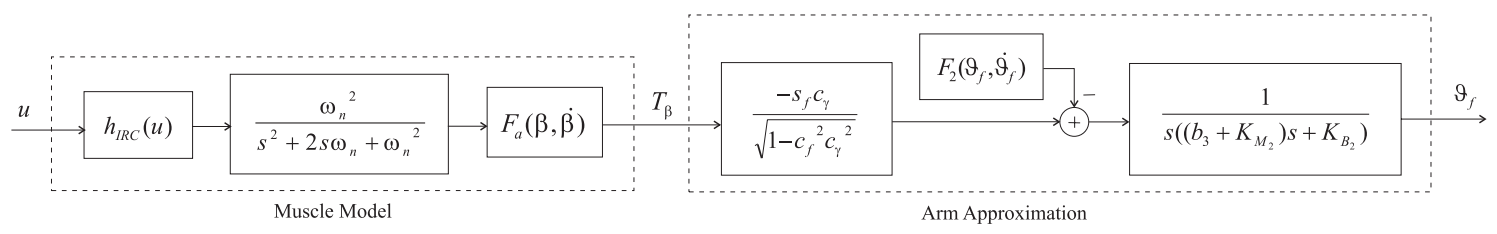

Fig. 5. Approximate model of stimulated human arm.

\section{CONTROL SCHEME}

A linearising controller will firstly be designed for the arm system and to this an ILC feedforward controller will be added. The results are from an unimpaired subject aged 57 years, and electromyographic (EMG) data has been used to ensure that they supplied no voluntary effort during the task.

\subsection{Linearising PD Controller}

The first component of the linearising controller is the inverse of the isometric recruitment function. Since the terms $-s_{f} c_{\gamma} / \sqrt{1-c_{f}^{2} c_{\gamma}^{2}}, F_{2}\left(\vartheta_{f}, \dot{\vartheta}_{f}\right)$ and $F_{a}(\beta, \dot{\beta})$ appearing in the arm model vary only slowly when the trajectories considered in this paper are followed perfectly (see Freeman et al. [2008]), the control action taken is to attempt to remove their effect in order to produce a system which approximates the linear activation dynamics in series with the linear arm dynamics (the transfer functions appearing in the left and right sub-systems respectively of Figure 5). To achieve this, the linearising controller with input $w$, is given by

$$
u=h_{I R C}^{-1}\left(\left(F_{2}\left(\vartheta_{f}, \dot{\vartheta}_{f}\right)+w\right) \frac{-\sqrt{1-c_{f}^{2} c_{\gamma}^{2}}}{s_{f} c_{\gamma} F_{a}(\beta, \dot{\beta})}\right)
$$

and the validity of this approach will now be investigated. For concision, attention is restricted to the case where $F_{2}(\cdot)$ and $F_{a}(\cdot)$ are functions of their first argument only. The system that is then formed is shown in Figure 6 in which

$$
g\left(\vartheta_{f}\right)=\frac{-s_{f} c_{\gamma} F_{a}\left(\beta\left(\vartheta_{f}\right)\right)}{\sqrt{1-c_{f}^{2} c_{\gamma}^{2}}}
$$

The linear activation dynamics can be represented by the state-

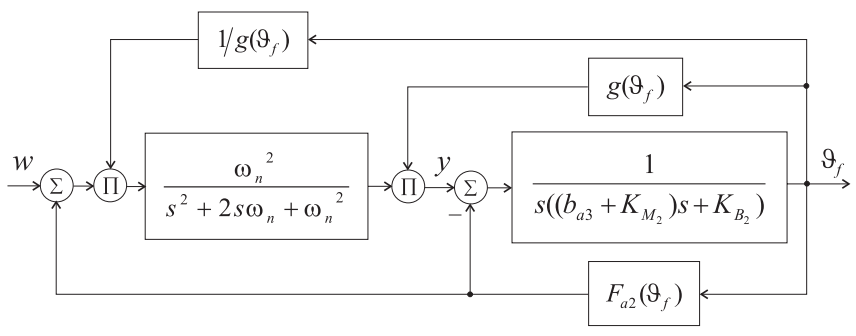

Fig. 6. Stimulated arm system and linearising controller.

space system $\left[\boldsymbol{A}_{m}, \boldsymbol{B}_{m}, \boldsymbol{C}_{m}\right]$, and the relationship between $w$ and $y$ is then given by

$\dot{\hat{\boldsymbol{x}}}_{m}(t)=\boldsymbol{A}_{m} \hat{\boldsymbol{x}}_{m}(t)+\boldsymbol{B}_{m}\left(\frac{\zeta(t)}{g\left(\vartheta_{f}(t)\right)}\right), \quad y(t)=\boldsymbol{C}_{m} \hat{\boldsymbol{x}}_{m}(t) g\left(\vartheta_{f}(t)\right)$

where $\zeta=w+F_{2}\left(\vartheta_{f}\right)$. The transformation $\boldsymbol{x}_{m}(t)=\hat{\boldsymbol{x}}_{m}(t) g\left(\vartheta_{f}\right)$ allows the system to absorb the effect of $g\left(\vartheta_{f}\right)$ to become

$$
\dot{\boldsymbol{x}}_{m}(t)=\left(\boldsymbol{A}_{m}+\boldsymbol{I} g_{1}(t)\right) \boldsymbol{x}_{m}(t)+\boldsymbol{B}_{m} \zeta(t), \quad y(t)=\boldsymbol{C}_{m} \boldsymbol{x}_{m}(t)
$$

in which $g_{1}(t)=\frac{\dot{g}\left(\vartheta_{f}(t)\right)}{g\left(\vartheta_{f}(t)\right)}$. The effect of $g(\cdot)$ is therefore to directly move the eigenvalues of its state transition matrix. Let the arm dynamics be represented by the state-space system $\left[\boldsymbol{A}_{p}, \boldsymbol{B}_{p}, \boldsymbol{C}_{p}\right]$ so that the relationship between $y$ and $\vartheta_{f}$ is given by

$$
\dot{\boldsymbol{x}}_{p}(t)=\boldsymbol{A}_{p} \boldsymbol{x}_{p}(t)+\boldsymbol{B}_{p}\left(y(t)-F_{a 2}\left(\vartheta_{f}\right)\right), \quad \vartheta_{f}(t)=\boldsymbol{C}_{p} \boldsymbol{x}_{p}(t)
$$

then linearising the complete system at $t=\bar{t}$ yields

$$
\begin{aligned}
\dot{\boldsymbol{X}} & =\left[\begin{array}{cc}
\boldsymbol{A}_{m}-\boldsymbol{I} \boldsymbol{C}_{p} \boldsymbol{A}_{p} \boldsymbol{x}_{p}(\bar{t}) g_{1}(\bar{t}) & \boldsymbol{W} \\
\boldsymbol{B}_{p} \boldsymbol{C}_{m} & \boldsymbol{A}_{p}-\boldsymbol{B}_{p} \boldsymbol{C}_{p} F_{2}^{\prime}\left(\vartheta_{f}(\bar{t})\right)
\end{array}\right] \boldsymbol{X} \\
& +\left[\begin{array}{ll}
0 & \boldsymbol{B}_{m}
\end{array}\right]^{T} \tilde{w}, \quad \tilde{\vartheta}_{f}=\left[\begin{array}{ll}
0 & \boldsymbol{C}_{m}
\end{array}\right] \boldsymbol{X}
\end{aligned}
$$

where $\boldsymbol{X}=\left[\begin{array}{ll}\tilde{\boldsymbol{x}}_{m}(t) & \tilde{\boldsymbol{x}}_{p}(t)\end{array}\right]^{T}$, and $\boldsymbol{W}=\boldsymbol{I} \boldsymbol{C}_{p} \boldsymbol{A}_{p}\left(\boldsymbol{x}_{m}(\bar{t}) g_{1}(\bar{t})+\right.$ $\left.\boldsymbol{x}_{p}(\bar{t}) \boldsymbol{C}_{p} \boldsymbol{x}_{m}(\bar{t})\left(g_{2}(\bar{t})-g_{1}(\bar{t})^{2}\right)\right)+\boldsymbol{B}_{m} \boldsymbol{C}_{p} \boldsymbol{F}_{2}^{\prime}\left(\vartheta_{f}(\bar{t})\right)$ in which $g_{2}(t)=\frac{g^{\prime \prime}\left(\vartheta_{f}(t)\right)}{g\left(\vartheta_{f}(t)\right)}$. If the derivatives of the $F_{2}\left(\vartheta_{f}\right)$ and $g\left(\vartheta_{f}\right)$ are zero for all $t$, this system corresponds with the desired relationship

$$
\frac{\vartheta_{f}}{w}(s)=\frac{\omega_{n}^{2}}{s^{2}+2 s \omega_{n}+\omega_{n}^{2}} \cdot \frac{1}{s\left(\left(b_{a 3}+K_{M_{2}}\right) s+K_{B_{2}}\right)}=G(s)
$$

For this to approximate the system behaviour when the derivatives are non-zero, trajectories must be chosen over which $g_{1}(t)$, $g_{2}(t)$ and $F_{2}^{\prime}(\cdot)$ are small. To examine whether the choice of trajectories and arm dynamics that are used in Section 6 correspond to a model that is well approximated by (21), simulations have been conducted in which its output is compared with that of the linearising controller applied to the full model shown in Figure 5. The applied input, $w$, has been chosen to result in approximate tracking of the more rapid of those demands used. The results are shown in Figure 7, and since the model outputs are in close agreement, they support the use of $G(s)$ to approximate the combined linearising controller and arm system. Care must be taken, however, to ensure that the control

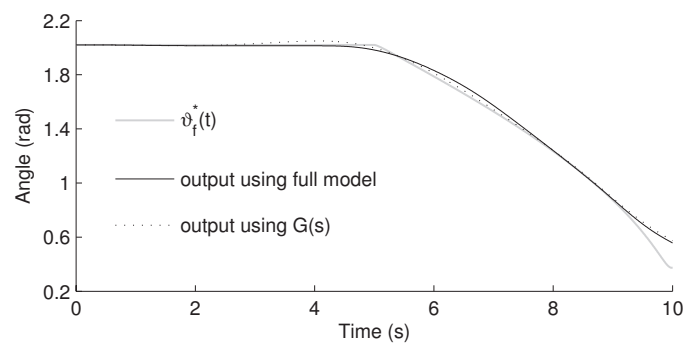

Fig. 7. Comparison of $G(s)$ with full arm model.

schemes designed using $G(s)$ are robust to the modelling error that exists.

A proportional derivative (PD) controller has been selected to supply the linearising controller input, $w$. Its transfer function is given by $C(s)$ and the resultant system is shown schematically in Figure 8. The level of stimulation that first produces a response from the triceps, $u_{m}$, is used to supply an offset so 


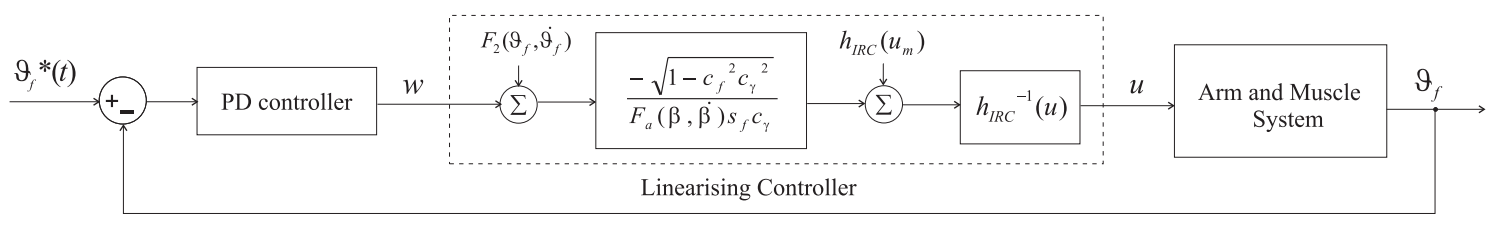

Fig. 8. Block diagram of feedback control system.

that the feedback system operates within the torque generating capabilities of the muscle. The closed-loop system is given by $P(s)=\frac{C(s) G(s)}{1+C(s) G(s)}$, and the feedback controller is tuned with an emphasis on robustness, since stability is of greater concern than accurate tracking. To illustrate the role of the end-effector dynamics on the system bandwidth, Bode plots of the closed-loop system are shown in Figure 9 for the ratios $\frac{b_{3}+K_{M_{2}}}{K_{B 2}}=0.03,0.07,0.4,0.8$ and 1.5 respectively. These have been created using the experimentally identified value of $\omega_{n}=$ $0.85 \pi$, and have been tuned using the standard Zeigler-Nichols rules. The corresponding closed-loop bandwidths are $\omega_{b}=$ $0.53 \mathrm{~Hz}, 0.45 \mathrm{~Hz}, 0.42 \mathrm{~Hz}, 0.23 \mathrm{~Hz}$ and $0.16 \mathrm{~Hz}$, and the models are termed $P_{0.53}, P_{0.45}, P_{0.42}, P_{0.23}$ and $P_{0.16}$. Alteration of $\frac{b_{3}+K_{M_{2}}}{K_{B 2}}$ cannot produce bandwidths much greater than these, and the principle objective is to make the task feel natural to the patient.
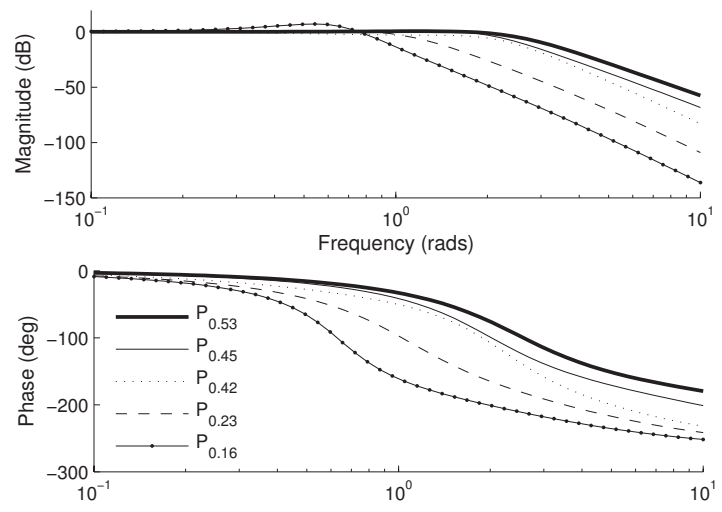

Fig. 9. Bode plots of closed-loop systems.

\subsection{Adjoint ILC}

The adjoint ILC algorithm is given in discrete form by

$$
v_{k+1}(z)=v_{k}(z)+\beta P^{*}(z) e_{k}(z)
$$

where $k$ is the trial number, $P^{*}(z)$ is the adjoint of the plant model, $e_{k}=\vartheta_{f}^{*}-\vartheta_{f, k}$, and $v_{k}$ replaces $\vartheta_{f}^{*}$ as the input to the system shown in Figure 8 (Furuta and Yamakita [1987]). The monotonic convergence criterion is given by

$$
\left|1-\beta P\left(e^{j \omega T_{s}}\right) P^{*}\left(e^{j \omega T_{s}}\right)\right|<1
$$

for $\omega$ up to the Nyquist frequency. The algorithm has been found to provide a high level of robustness to plant uncertainty (Freeman et al. [2005]). Figure 10 shows the monotonic convergence criterion using $P_{0.45}$ for a variety of $\beta$ values. For frequencies where the closed-loop system has a gain close to unity, approximate convergence will occur in a single trial for values of $\beta$ close to one. In practice, however, $\beta$ is chosen to be significantly lower in order to increase the system robustness at the expense of the convergence rate.

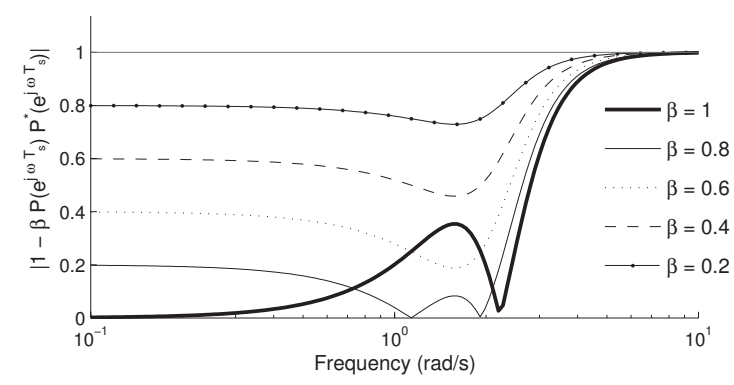

Fig. 10. Monotonic convergence criterion for $P_{0.45}$.

\section{EXPERIMENTAL RESULTS}

The subject's arm model was first identified using tests described in Freeman et al. [2008]. In particular FES was applied to the triceps using a ramp signal, and $h_{I R C}(u)$ and $h_{L A D}(t)$ were found using deconvolution and an non-linear optimisation procedure. Stimulation sequences and kinematic trajectories were then applied to the arm and an LMS optimisation was used to yield the remaining model parameters. Figure 11 shows the shape of the reference used to produce the results in this paper. It is set at an angle of $20^{\circ}$ from the $y$ axis and extends the subject's arm from $55 \%$ to $95 \%$ of their maximum reach. The

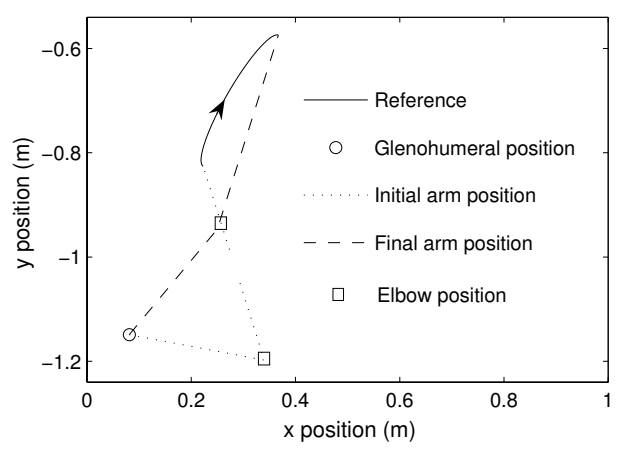

Fig. 11. Reference trajectory and position of subject tested.

trajectory consists of a 5 second waiting period and a 7.5 second movement along the reference at a constant speed. Before each trial began, the subject's arm was moved to the initial position by the robot and then released when the trajectory started. The subject was not shown the trajectory before or during the test and EMG recordings were inspected to ensure no voluntary effort was exerted.

The identified value of $b_{3}$ was $0.27 \mathrm{Kgm}^{2}$, and, following the discussion in Section 5.1, the values of $K_{B_{2}}$ and $K_{M_{2}}$ were set at $8 \mathrm{Nm} / \mathrm{rads}^{-1}$ and $0.29 \mathrm{Nm} / \mathrm{rads}^{-2}$ respectively and the controller gains then tuned to produce a closed-loop system with model $P_{0.45}$. Figure 12 shows results using the adjoint algorithm using $\beta=0.2$, and, in order to examine the robustness of the algorithm, the other models described in Section 5.1 have also been used. It can be seen that the algorithm is capable of 


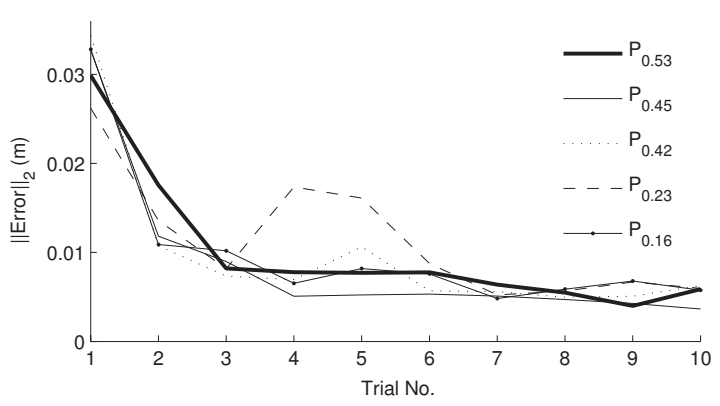

Fig. 12. Adjoint ILC results for the slow trajectory with $\beta=0.2$ and various plant models.

exhibiting robustness to significant model uncertainty. Use of the actual plant model, $P_{0.45}$, results in convergence to an $l_{2}$ error norm of less than $5 \mathrm{~mm}$ within 4 trials and this level of error is maintained over the remaining trials. Figure 13 shows tracking results over the first 5 trials and highlights the monotonicity of convergence. Figure 14 shows the corresponding

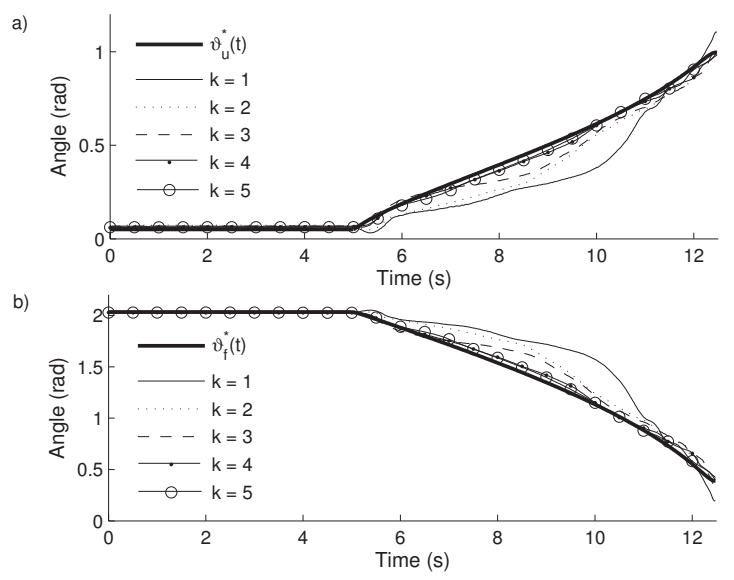

Fig. 13. Experimental tracking of a) $\vartheta_{u}^{*}(t)$ and b) $\vartheta_{f}^{*}(t)$ using adjoint ILC for the slow trajectory with $\beta=0.2$.

stimulation input and ILC update. It can be seen from a) that
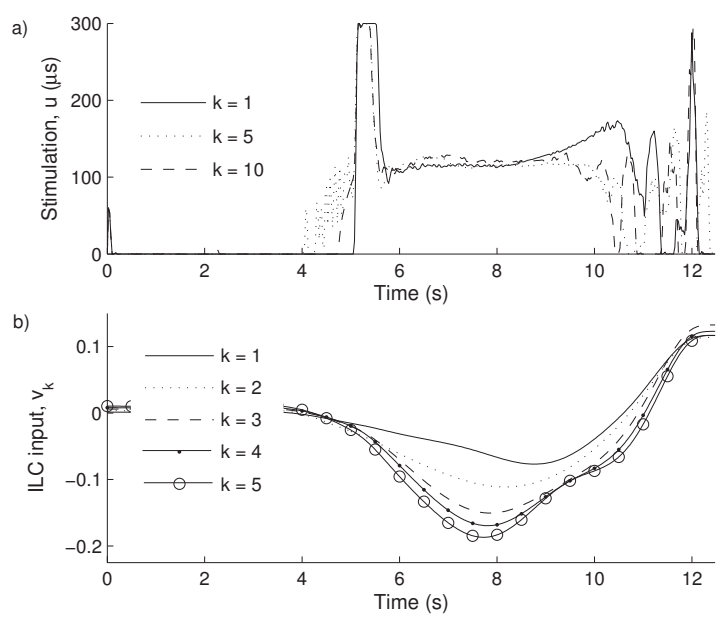

Fig. 14. Experimental a) stimulation and b) updated input results using adjoint ILC for the slow trajectory with $\beta$ $=0.2$.

ILC causes the stimulation to decrease from the level observed initially, and leads to its application during the initial 5 second waiting period. Figure $14 \mathrm{~b}$ ) illustrates the convergence of the ILC update.

\section{CONCLUSIONS}

The feasibility of tracking reaching trajectories using FES applied to the triceps muscle in combination with robotic assistance has been investigated. Results have shown that a high level of tracking performance is possible in combination with a high level of robustness. The results confirm the efficacy of a combined robotic assistance and stimulation system that has been designed, and provide justification for it to be subsequently used by stroke patients for rehabilitation.

\section{REFERENCES}

R. Baratta and M. Solomonow. The dynamic response model of nine different skeletal muscles. IEEE Transactions on Biomedical Engineering, 37(3):243-251, March 1990.

J. H. Burridge and M. Ladouceur. Clinical and therapeutic applications of neuromuscular stimulation: A review of current use and speculation into future developments. Neuromodulation, 4(4):147-154, 2001.

J. E. Colgate and N. Hogan. Robust control of dynamically interacting systems. International Journal of Control, 48(1): 65-88, 1988.

R. Davoodi and B. J. Andrews. Fuzzy logic control of FES rowing exercise in paraplegia. IEEE Transactions on Biomedical Engineering, 51(3):541-543, 2004.

H. Dou, K. K. Tan, T. H. Lee, and Z. Zhou. Iterative learning control of human limbs via functional electrical stimulation. Control Engineering Practice, 7:315-325, 1999.

C. T. Freeman, P. L. Lewin, and E. Rogers. Experimental evaluation of iterative learning control algorithms for nonminimum phase plants. International Journal of Control, 78 (11):826-846, July 2005.

C. T. Freeman, A. M. Hughes, J. H. Burridge, P. H. Chappell, P. L. Lewin, and E. Rogers. An experimental facility for the application of iterative learning control as an intervention aid to stroke rehabilitation. Measurement + Control: The Journal of the Institute of Measurement and Control, 40(1): 20-23, February 2007.

C. T. Freeman, A. M. Hughes, J. H. Burridge, P. H. Chappell, P. L. Lewin, and E. Rogers. A model of the upper extremity using surface FES for stroke rehabilitation. Submitted to: Journal of Biomechanical Engineering, 2008.

K. Furuta and M. Yamakita. The design of learning control systems for multivariable systems. In Proceedings of the IEEE International Symposium on Intelligent Control, pages 371-376, Philadelphia, Pennsylvania, 1987.

N. Lan, H. Q. Feng, and P. E. Crago. Neural network generation of muscle stimulation patterns for control of arm movements. IEEE Transactions on Rehabilitation Engineering, 2(4):213224, December 1994.

J. Mant, D. Wade, and S. Winner. Health care needs assessment: stroke. In A. Stevens, J. Raftery, J. Mant, and S. Simpson, editors, Health care needs assessment: the epidemiologically based needs assessment reviews. Oxford: Radcliffe Medical Press, 2004.

K. L. Moore. Iterative Learning Control for Deterministic Systems. Springer-Verlag, Berlin, 1992.

G. Shue, P. E. Crago, and H. J. Chizeck. Muscle-joint models incorporating activation dynamics, moment-angle, and moment-velocity properties. IEEE Transactions on Biomedical Engineering, 42(2):213-223, February 1995. 